\title{
Radiosensitivity of Oncological Patients
}

E. Novikova and I. Albitskiy

Scientific Center of Roentgenology \&3 Radiology, 86, Profsouznaya Str., 117997 Moscow, Russian Federation

snigiryova@rncrr.ru

One of the most serious problems of radiotherapy is complications which occur in normal tissue after irradiation. Very often damage of normal tissues caused by irradiation is an obstacle for continuation of treatment course. Therefore in the present paper damage of normal tissues of oncological patients with different tumor types after radiotherapy course have been studied. Damage of normal tissues was clinically registered; cytogenetic and molecular-biological changes in blood cells were studied in radiobiological researches. The level of chromosome aberrations (cytogenetic method) and DNA damage (comet assay, single-strand breaks of DNA) was established before and after radiotherapy. We showed that the level of the chromosome aberrations induced by an additional irradiation of blood cells in vitro in the group of patients without sever acute reactions was higher than in the group of patients with irradiation damage of normal tissues. The level of the residual damage defined by comet assay in 20 minutes of reparation after an additional irradiation of blood cells in vitro in the group of oncological patients after radiotherapy have a tendency to increase. The patients who have received damage of normal tissues during irradiation (acute reactions) are characterized by slower repair kinetics in comparison with the patients without this damage. However, characteristics of repair capacity of separate individuals can essentially differ. 